\title{
Desflurane-induced and ischaemic postconditioning against myocardial infarction are mediated by Pim-1 kinase
}

\author{
J. Stumpner ${ }^{1}$, T. M. Smul ${ }^{1}$, A. Redel ${ }^{1,2}$, T. Hilz ${ }^{1}$, T. Tischer-Zeitz ${ }^{1}$, H. Eisenbarth ${ }^{3}$, M. A. Schick ${ }^{1}$, F. KehL ${ }^{4}$, \\ N. RoEWER ${ }^{1}$ and M. LANGE ${ }^{1,5}$ \\ ${ }^{1}$ Department of Anaesthesia and Critical Care, University of Würzburg, Würzburg, Germany, ${ }^{2}$ Department of Anesthesia, University of \\ Regensburg, Regensburg, Germany, ${ }^{3}$ Department of Forensic Psychiatry and Psychotherapy, University of Regensburg, Regensburg, Germany, \\ ${ }^{4}$ Department of Anesthesiology and Critical Care, Hospital of Karlsruhe, Karlsruhe, Germany and ${ }^{5}$ Department of Anesthesia and Critical Care \\ Medicine, Mathias-Spital Rheine, Germany
}

Background: Anaesthetic-induced (APOST) and ischaemic postconditioning (IPOST) against myocardial infarction are mediated via phosphatidylinositol-3-kinase/Akt. Pim-1 kinase is acting downstream of Akt and has recently been demonstrated to enhance cardiomyocyte survival. We tested the hypothesis that both APOST and IPOST are mediated by Pim-1 kinase.

Methods: Pentobarbital-anaesthetized male C57BL/6 mice were subjected to 45-min coronary artery occlusion (CAO) and 3-h reperfusion. Animals received either no intervention, the Pim-1 kinase inhibitor II $(10 \mu \mathrm{g} / \mathrm{g}$ intraperitoneally) or its vehicle dimethy sulfoxide $(10 \mu \mathrm{l} / \mathrm{g}$ intraperitoneally). Three minutes prior to the end of $\mathrm{CAO}, 1.0$ minimum alveolar concentration desflurane was administered for $18 \mathrm{~min}$ alone or in combination with Pim-1 kinase inhibitor II. IPOST was induced by three cycles of each 10-s ischaemia/reperfusion, and animals received either IPOST alone or in combination with Pim-1 kinase inhibitor II. Infarct size was determined with triphenyltetrazolium chloride and area at risk with Evans blue. Protein expression of Pim-1 kinase, Bad, phospho-Bad ${ }^{\text {Ser112 }}$ and B-cell lymphoma 2 was determined using Western immunoblotting analysis.

Results: Infarct size in control animals (CON) was $46 \pm 3 \%$. Dimethylsulfoxide $(47 \pm 3 \%)$ and Pim-1 kinase inhibitor II $(44 \pm 5 \%)$ did not significantly reduce infarct size. Desflurane $\left(16 \pm 2 \%{ }^{*}{ }^{*} P<0.05\right.$ vs. CON) and IPOST $\left(21 \pm 2 \%{ }^{*}\right)$ significantly reduced infarct size compared with CON. Inhibition of Pim-1 kinase abolished desflurane-induced postconditioning $(46 \pm 4 \%)$ and IPOST (44 $\pm 5 \%$ ). Western blot analysis revealed that only desflurane enhances phosphorylation of Bad at serine 112 that was abrogated by Pim-1 kinase inhibitor II.

Conclusion: These data suggest that Pim-1 kinase mediates both desflurane-induced postconditioning and IPOST in mice.

Accepted for publication 11 January 2012

() 2012 The Authors

Acta Anaesthesiologica Scandinavica

(c) 2012 The Acta Anaesthesiologica Scandinavica Foundation
A LTHOUGH early myocardial reperfusion is regarded the most effective strategy to treat acute myocardial ischaemia, considerable evidence has accumulated that myocardial reperfusion itself causes cardiomyocyte death. ${ }^{1,2}$ This is known as lethal reperfusion injury. Myocardial reperfusion is estimated to cause $50 \%$ of myocardial infarct size (IS) as a result of ischaemia/reperfusion injury. ${ }^{2}$ Further support to this concept was provided by the study of Zhao et al., who demonstrated that three cycles of each 30-s reperfusion/ischaemia immediately after coronary artery occlusion (CAO) reduced myocardial IS up to $40 \%$ in dogs. ${ }^{3}$ This phenomenon was termed ischaemic postconditioning
(IPOST). ${ }^{3}$ IPOST has been demonstrated to reduce myocardial IS among others in dogs, ${ }^{3}$ rabbits ${ }^{4}$ and mice. $^{5-7}$ In humans, IPOST has been shown to reduce myocardial IS as assessed 6 months after acute myocardial infarction and to improve left ventricular ejection fraction after 1 year. ${ }^{8}$

Cardioprotective properties of volatile anaesthetics during early reperfusion are known for more than a decade since the first description of Schlack's group. ${ }^{9}$ Later, this phenomenon was termed anaesthetic-induced postconditioning (APOST). ${ }^{10}$ APOST reduces myocardial IS, 5,10,11 improves left ventricular function after myocardial ischaemia ${ }^{12}$ and has been demonstrated to occur in humans. ${ }^{13}$ 


\section{J. Stumpner et al.}

Isoflurane, sevoflurane and desflurane (DES) are equally effective to reduce myocardial IS in mice in vivo. ${ }^{5}$

Both IPOST and APOST afford their cardioprotective properties via reduction of reperfusion injury and are mediated via phosphatidylinositol-3-kinase (PI3K)/ $\mathrm{Akt}^{10,14,15}$ as a part of the reperfusion injury salvage kinase (RISK) pathway. ${ }^{16}$

The serine/threonine kinase Pim- 1 belongs to the family of calcium/calmodulin-dependent protein kinases (CaMK). ${ }^{17}$ DES-induced postconditioning is mediated by CaMKII in rabbits in vivo. ${ }^{11}$ Pim-1 kinase has recently been described to act downstream of PI3K/Akt in cardiac myocytes. ${ }^{18}$ Geneticinduced overexpression of Pim-1 confers antiapoptotic $^{18}$ and antihypertrophic effects, ${ }^{19}$ and reduces myocardial IS. ${ }^{18}$ Moreover, genetic engineering of cardiac progenitor cells with Pim-1 kinase has been described to improve cellular viability, proliferation and regeneration after acute myocardial infarction. ${ }^{20}$ More recently, our group demonstrated a crucial role for Pim-1 kinase in both DES-induced and ischaemic preconditioning (IPC) against myocardial infarction. ${ }^{21}$

However, whether Pim-1 kinase is involved in APOST and IPOST against myocardial infarction has not been investigated to date. Therefore, we tested the hypothesis that APOST and IPOST are mediated via Pim-1 kinase in mice in vivo.

\section{Methods}

\section{Ethical approval}

All experimental procedures used in this investigation were reviewed and approved by the Animal Care and Use Committee of the Government of Lower Franconia, Bavaria, Germany. All experiments were in accordance with the Guide for the Care and Use of Laboratory Animals, ${ }^{22}$ and conformed to the Guiding Principles in the Care and Use of Animals of the American Physiological Society.

\section{Instrumentation and surgical procedures}

Male C57BL/ 6 mice (10-12 weeks old) were purchased from Harlan laboratories (Horst, the Netherlands). Animals were housed under controlled conditions $\left(22{ }^{\circ} \mathrm{C}, 55-65 \%\right.$ humidity, 12-h light-dark cycle), and were allowed free access to water and a standard laboratory chow. Instrumentation and surgical procedures were performed, as described previously. ${ }^{23}$ Briefly, mice were anaesthetized with an intraperitoneal injection of $60 \mu \mathrm{g} / \mathrm{g}$ sodium pento- barbital (Merial, Hallbergmoos, Germany), and repeated intraperitoneal injections were given as needed to maintain anaesthesia. Rectal temperature was maintained at $37.0 \pm 0.1^{\circ} \mathrm{C}$ using a servocontrolled heating-pad (FMI, Seeheim, Germany). After intubation of the trachea, animals were ventilated with 50\%/50\% air-oxygen mixture using a small rodent ventilator (SAR-P 830, CWE, Inc., Ardmore, PA, USA) operating in pressurecontrolled mode. A three-lead needle-probe electrocardiogram (ECG) was attached to continuously monitor heart rate and ST segment elevation. Salinefilled polyethylen (PE) catheters were placed into the right common carotid artery for measurement of mean arterial blood pressure and into the right jugular vein for continuous fluid administration $(20 \mu \mathrm{l} / \mathrm{g} / \mathrm{h})$. A left thoracotomy at the fourth intercostal space was performed, and the left anterior descending coronary artery (LAD) was exposed. $\mathrm{CAO}$ was achieved using the hanging weight system $^{23,24}$ and was verified by ECG ST segment elevation and paleness of the myocardial area at risk (AAR). Adequate reperfusion was verified by epicardial hyperaemia and reversion of ECG changes.

\section{Experimental protocol}

Animals were randomly assigned to seven groups to investigate the role of Pim-1 kinase in ischaemic and DES-induced postconditioning. Group size was $n=8$ in each group. The experimental protocol is illustrated in Figure 1.

After completion of surgical procedures, all mice were allowed a 30-min equilibration period. Myocardial ischaemia was induced by $45-\mathrm{min}$ CAO followed by $3 \mathrm{~h}$ of reperfusion. Control animals (CON) received no additional treatment. In group 2 [dimethyl sulfoxide (DMSO)], the vehicle DMSO $(10 \mu \mathrm{l} / \mathrm{g})$ was injected intraperitoneally $5 \mathrm{~min}$ prior to the end of CAO. In group 3 [Pim-1 kinase inhibitor II (PIM-Inh.II)], the selective ${ }^{25}$ Pim-1 kinase inhibitor 2-hydroxy-3-cyano-4-phenyl-6-(3-bromo6-hydroxyphenyl)pyridine (PIM-Inh.II, $10 \mu \mathrm{g} / \mathrm{g}$, dissolved in DMSO; Merck, Darmstadt, Germany) was administered intraperitoneally $5 \mathrm{~min}$ prior to the end of CAO. DES was administered at a concentration of 1.0 minimum alveolar concentration (MAC, $7.5 \mathrm{Vol} \%{ }^{26}$ ) for 18 min starting 3 min prior to the onset of reperfusion. This type of administration was chosen because the first minute of reperfusion after CAO is most probably critical for postconditioning's success. ${ }^{27}$ Initiation of DES administration before reperfusion guaranteed an effective level of 1.0 MAC DES with the onset of reperfusion. 


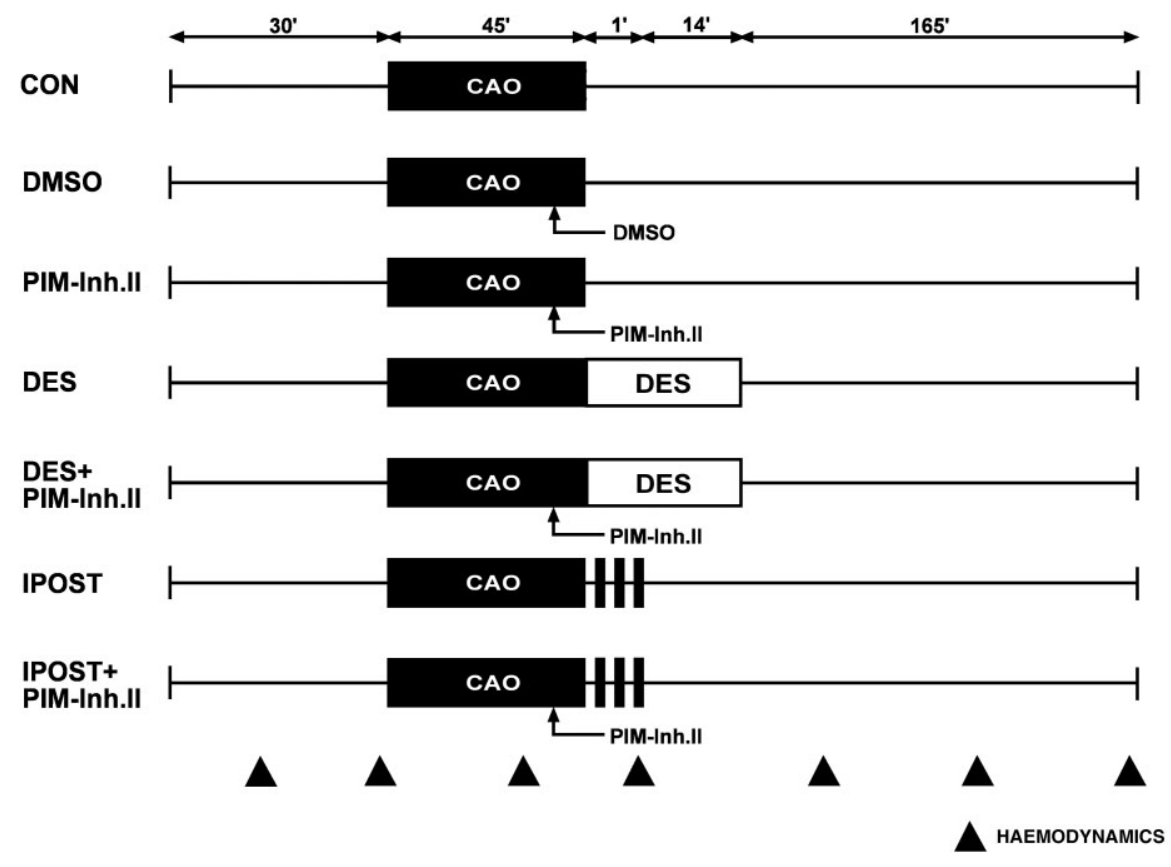

Fig. 1. Schematic diagram illustrating the experimental protocol of the study. CAO, coronary artery occlusion; CON, control group; DMSO, dimethyl sulfoxide $(10 \mu \mathrm{l} / \mathrm{g}$ ) intraperitoneal $5 \mathrm{~min}$ prior to the onset of reperfusion; PIM-Inh.II, Pim-1 kinase inhibitor II $(10 \mu \mathrm{g} / \mathrm{g})$ intraperitoneal 5 min prior to the onset of reperfusion; DES, desflurane (1.0 minimum alveolar concentration DES for $18 \mathrm{~min}$ starting $3 \mathrm{~min}$ prior to the end of $\mathrm{CAO}$ ); DES+PIM-Inh.II, PIM-Inh.II $(10 \mu g / g)$ intraperitoneal $5 \mathrm{~min}$ prior to the end of CAO and DES (1.0 minimum alveolar concentration for $18 \mathrm{~min})$; IPOST, ischaemic postconditioning (three cycles of each $10 \mathrm{~s}$ ischaemia/reperfusion immediately after the end of CAO); IPOST+PIM-Inh.II, PIM-Inh.II $(10 \mu \mathrm{g} / \mathrm{g}) 5 \mathrm{~min}$ prior to IPOST. Triangles indicate time points of analysis of haemodynamic parameters, as presented in Table 1.

Animals of group 5 received PIM-Inh.II in combination with DES (DES+PIM-Inh.II). IPOST was induced by three cycles of each 10-s ischaemia and reperfusion immediately after CAO. In group 7, animals received PIM-Inh.II in combination with IPOST (IPOST+PIM-Inh.II).

\section{Measurement of myocardial IS}

Myocardial IS and AAR were determined using methods described previously. ${ }^{23}$ Briefly, after $3 \mathrm{~h}$ of reperfusion, the LAD was re-occluded and $1 \mathrm{ml}$ Evans blue $(0.1 \mathrm{~g} / \mathrm{ml}$, Sigma-Aldrich, Taufkirchen, Germany) was slowly injected into the carotid artery. After intraperitoneal injection of a lethal dose of sodium pentobarbital $(150 \mu \mathrm{g} / \mathrm{g})$, the heart was rapidly excised. The left ventricle was separated and cut into 7-8 transversal slices of 1-mm thickness. Slices were incubated in 2,3,5-triphenyltetrazolium chloride $(20 \mathrm{mg} / \mathrm{ml})$ for $30 \mathrm{~min}$ at $37^{\circ} \mathrm{C}$. After overnight fixation in $10 \%$ formaldehyde, slices were weighted and digitally photographed. Photographs were analysed using Adobe Photoshop CS 8.0.1 (Adobe Systems, Inc., San Jose, CA, USA), and the normal zone, AAR and IS were determined gravitoplanimetrically by an investigator blinded to the experimental protocol. Animals with an AAR of less than $20 \%$ were excluded from the study.

\section{Western immunoblotting}

In a second set of experiments, myocardial tissue was extracted after $1 \mathrm{~h}$ of reperfusion to investigate effects on the expression of Pim-1 kinase, Bad and B-cell lymphoma 2 (Bcl-2), and to investigate phosphorylation of Bad at serine 112 as a surrogate for Pim-1 activity using Western immunoblotting technique.

Mice ( $n=4$ in each group) received saline (CON), PIM-Inh.II, DES alone or in combination with PIM-Inh.II (DES+PIM-Inh.II), and IPOST alone (IPOST), or in combination with PIM-Inh.II (IPOST+PIM-Inh.II). One hour after the onset of reperfusion, hearts were rapidly excised, and the left ventricle was shock frozen in liquid nitrogen and stored at $-80^{\circ} \mathrm{C}$ until further usage. The samples were homogenized in ice-cold radioimmunoprecipitation assay buffer [phosphatebuffered saline (PBS) $\left(\mathrm{Na}_{2} \mathrm{HPO}_{4}, \mathrm{NaH}_{2} \mathrm{PO}_{4}, \mathrm{NaCl}\right.$, $\mathrm{H}_{2} \mathrm{O}$; ph 7.4), $1 \%$ Igepal CA-630, $0.5 \%$ sodium desoxycholic acid, $0.1 \%$ sodium dodecylsulfate polyacrylamide; for phospho-samples: protease inhibitor, $\mathrm{NaF} 20 \mathrm{mM}$, sodium vanadate $1 \mathrm{mM}$ in addition) and centrifuged at $12,000 \mathrm{~g}$ at $4{ }^{\circ} \mathrm{C}$. Cytosolic and particulate cell fractions were left unseparated for further analysis.

Proteins were loaded on $15 \%$ polyacrylamide sodium dodecylsulfate polyacrylamide gels and were subsequently blotted on nitrocellulose membranes (Protran, Whatman $\mathrm{GmbH}$, Dassel, Germany). Nonspecific background was blocked using $2.5 \%$ nonfat milk powder combined with $2.5 \%$ albumin powder from bovine serum in PBS-Tween 20 ( $1 \mathrm{~h}$ at room temperature). Membranes were then 


\section{J. Stumpner et al.}

incubated with the following antibodies: antiPim-1 kinase 1:100 (Santa Cruz Biotechnology, Santa Cruz, CA, USA), antiBad 1:100 (Abcam, Cambridge, Great Britain), antiphospho-Bad ${ }^{\text {Ser112 } 1: 500}$ (Cell Signaling, Danvers, MA, USA), antiBcl-2 $1: 500$ (Abcam), mouse antiglyceraldehyde-3phosphate dehydrogenase (Millipore, Billerica, MA, USA) and mouse anti- $\alpha \mathrm{B}$-crystallin $1: 3000$ (Assay Designs, Ann Arbor, MI, USA), respectively.

The protein bands were detected using enhanced chemiluminescent ${ }^{\circledR}$ detection reagent (GE Healthcare, Buckinghamshire, UK) and visualized on an X-ray film. The films were scanned and optical density was determined using ImageJ software (NIH, Bethesda, MD, USA). Optical density of the target protein was normalized to its loading control expression. Glyceraldehyde-3-phosphate dehydrogenase served as the loading control for Bad, phospho-Bad ${ }^{\mathrm{Ser112}}$ and $\mathrm{Bcl}-2$, and $\alpha \mathrm{B}-$ crystallin served as the loading control for Pim-1 kinase, respectively. The control group ratio was set as $100 \%$.

\section{Data acquisition and statistical analysis}

ECG, systemic haemodynamic parameters and body temperature were continuously recorded and analysed on a personal computer (Fujitsu Siemens, Augsburg, Germany) using a haemodynamic data acquisition and analysis software (Notocord ${ }^{\circledR}$ hem 3.5, Croissy-sur-Seine, France).

Concluding from other studies on the same experimental model, ${ }^{21,23,28}$ we expected a myocardial IS of $50 \%$ (IS/AAR). Power analysis revealed a group size of $n=8$ to detect a reduction in means of $15 \%$ with a power of 0.8 at a $\alpha$-level of 0.05 . Statistical analyses were done by analysis of variance (ANOVA), which were based on two-tailed F-tests for comparison of components of the factors' total deviation. Analyses for body weight, left ventricle weight, left ventricle weight/body weight, AAR, IS, IS/left ventricle and AAR/left ventricle were performed using one-way ANOVA, including the factor treatment (CON vs. DMSO vs. PIM-Inh.II vs. DES vs. DES+PIM-Inh.II vs. IPOST vs. IPOST+PIMInh.II) and post hoc Duncan's test for significant main effects and interactions. Analysis for densitometry was also performed using one-way ANOVA including the factor treatment (CON vs. PIM-Inh.II vs. DES vs. DES+PIM-Inh.II vs. IPOST vs. IPOST+PIM-Inh.II) and post hoc Duncan's test for significant main effects and interactions. Analysis of haemodynamic data was performed by a $7 \times 7$ ANOVA for repeated measures, including the between factor treatment (CON vs. DMSO vs. PIMInh.II vs. DES vs. DES+PIM-Inh.II vs. IPOST vs. IPOST+PIM-Inh.II) and the within factor time point (baseline vs. PreCAO vs. CAO vs. POST vs. reperfusion $60 \mathrm{~min}$ vs. reperfusion $120 \mathrm{~min}$ vs. reperfusion $180 \mathrm{~min}$ ). In case of any significant main effects or interactions, post hoc one-way ANOVAs were conducted for each group and each time point, where a Bonferroni correction of $\alpha$ was performed. Statistical analysis of data was performed using SPSS 16.0 software (The Apache Software Foundation, Forest Hill, MD, USA). Changes in means were considered statistically significant when $P<0.05$. Data are presented as mean \pm standard error of the mean.

\section{Results}

A total of 86 mice were included to the study. Sixty-two mice were assigned to the ischaemiareperfusion experiments to obtain 56 successful experiments. Six animals were excluded because of pump failure during CAO (one in the DMSO group, one in the PIM-Inh.II group, one in the DES+PIMInh.II group, one in the IPOST group, two in the IPOST+PIM-Inh.II group). Twenty-four mice were used to measure protein expression using Western immunoblotting technique.

\section{Haemodynamic parameters, AAR}

Haemodynamic parameters at baseline and AAR were not different among groups (Tables 1 and 2). MAP was significantly decreased compared with baseline values during CAO in two groups (DMSO and DES). In the PIM-Inh.II group, MAP was significantly decreased compared with the control group after $120 \mathrm{~min}$ of reperfusion.

\section{Myocardial IS}

Myocardial IS (IS/AAR) was $46 \% \pm 3 \%$ in control group (Fig. 2). PIM-Inh.II ( $44 \% \pm 5 \%)$ alone and its vehicle DMSO $(47 \% \pm 3 \%)$ alone did not reduce myocardial IS. A 15-min administration of 1.0 MAC DES during early reperfusion (DES; $16 \% \pm 2 \%$; $P<0.05$ vs. control group) significantly reduced myocardial IS compared with control group. Inhibition of Pim-1 kinase using PIM-Inh.II completely abolished DES-induced postconditioning (DES+PIM-Inh.II; 46\% $\pm 4 \%$ ). IPOST induced by three cycles of each 10-s ischaemia and reperfusion reduced myocardial IS to $21 \% \pm 2 \%$ (IPOST; $P<0.05$ vs. control group, PIM-Inh.II and IPOST+PIM-Inh.II). Similar to DES-induced post- 
Table 1

\begin{tabular}{|c|c|c|c|c|c|c|c|}
\hline \multirow[t]{2}{*}{$\mathrm{HR}\left(\mathrm{min}^{-1}\right)$} & \multirow[t]{2}{*}{$\mathrm{BL}$} & \multirow[t]{2}{*}{ PreCAO } & \multirow[t]{2}{*}{ CAO } & \multirow[t]{2}{*}{ POST } & \multicolumn{3}{|c|}{ Reperfusion } \\
\hline & & & & & $60 \min$ & $120 \mathrm{~min}$ & $180 \mathrm{~min}$ \\
\hline $\begin{array}{l}\text { MAP (mmHg) } \\
\text { CON } \\
\text { DMSO } \\
\text { PIM-Inh.II } \\
\text { DES } \\
\text { DES+PIM-Inh.II } \\
\text { IPOST } \\
\text { IPOST+PIM-Inh.II }\end{array}$ & $\begin{array}{l}72 \pm 3 \\
71 \pm 5 \\
70 \pm 5 \\
69 \pm 2 \\
73 \pm 2 \\
71 \pm 5 \\
74 \pm 4\end{array}$ & $\begin{array}{l}66 \pm 5 \\
70 \pm 6 \\
67 \pm 5 \\
68 \pm 2 \\
68 \pm 3 \\
66 \pm 4 \\
71 \pm 4\end{array}$ & $\begin{array}{l}60 \pm 2 \\
63 \pm 5^{\star} \\
58 \pm 6 \\
58 \pm 3^{*} \\
67 \pm 2 \\
62 \pm 3 \\
63 \pm 4\end{array}$ & $\begin{array}{l}70 \pm 4 \\
67 \pm 7 \\
58 \pm 6 \\
63 \pm 4 \\
70 \pm 2 \\
67 \pm 3 \\
60 \pm 4\end{array}$ & $\begin{array}{l}71 \pm 4 \\
70 \pm 5 \\
59 \pm 3 \\
65 \pm 4 \\
64 \pm 4 \\
68 \pm 4 \\
66 \pm 3\end{array}$ & $\begin{array}{l}68 \pm 4 \\
67 \pm 4 \\
54 \pm 1^{\dagger} \\
64 \pm 3 \\
64 \pm 3 \\
65 \pm 3 \\
68 \pm 4\end{array}$ & $\begin{array}{l}66 \pm 6 \\
64 \pm 3 \\
56 \pm 3 \\
68 \pm 3 \\
72 \pm 6 \\
66 \pm 5 \\
67 \pm 4\end{array}$ \\
\hline
\end{tabular}

Data are mean \pm SEM.

Data were analysed during baseline period (BL), before (PreCAO) and during (CAO) CAO, during early reperfusion period (POST) and 60,120 and 180 min after the onset of reperfusion, as indicated by triangles in Figure 1.

*Significantly $(P<0.05)$ different from BL.

†Significantly $(P<0.05)$ different from CON.

$\mathrm{HR}$, heart rate; MAP, mean arterial pressure; CON, control; DMSO, dimethyl sulfoxide; PIM-Inh.II, Pim-1 kinase inhibitor II; DES, desflurane; IPOST, ischaemic postconditioning; CAO, coronary artery occlusion; SEM, standard error of the mean.

Table 2

\begin{tabular}{|c|c|c|c|c|c|c|c|c|}
\hline & $n$ & $\mathrm{BW}[\mathrm{g}]$ & $\mathrm{LV}$ [mg] & LV/BW [\%] & AAR [mg] & IS [mg] & IS/LV [\%] & AAR/LV [\%] \\
\hline $\mathrm{CON}$ & 8 & $25.4 \pm 0.7$ & $70.3 \pm 2.8$ & $0.28 \pm 0.01$ & $25.1 \pm 2.6$ & $11.4 \pm 1.2$ & $16.0 \pm 1.2$ & $35.3 \pm 2.6$ \\
\hline DMSO & 8 & $26.6 \pm 1.0$ & $77.3 \pm 3.1$ & $0.29 \pm 0.01$ & $25.0 \pm 2.1$ & $11.9 \pm 1.3$ & $15.4 \pm 1.6$ & $32.5 \pm 2.6$ \\
\hline PIM-Inh.II & 8 & $26.8 \pm 1.4$ & $75.9 \pm 5.2$ & $0.28 \pm 0.01$ & $23.5 \pm 1.8$ & $10.5 \pm 1.7$ & $13.7 \pm 1.7$ & $31.1 \pm 1.5$ \\
\hline DES & 8 & $24.5 \pm 1.2$ & $68.7 \pm 5.4$ & $0.28 \pm 0.01$ & $21.6 \pm 2.3$ & $3.5 \pm 0.7^{\star \dagger}$ & $5.1 \pm 0.9^{* \dagger}$ & $31.3 \pm 1.9$ \\
\hline DES+PIM-Inh.II & 8 & $25.9 \pm 1.1$ & $76.6 \pm 4.2$ & $0.30 \pm 0.01$ & $26.7 \pm 2.8$ & $12.1 \pm 1.7$ & $16.2 \pm 2.7$ & $36.0 \pm 5.1$ \\
\hline IPOST & 8 & $26.0 \pm 0.4$ & $72.9 \pm 2.2$ & $0.28 \pm 0.01$ & $21.6 \pm 2.5$ & $4.3 \pm 0.5^{\star \ddagger}$ & $6.0 \pm 0.6^{\star \ddagger}$ & $29.6 \pm 3.2$ \\
\hline IPOST+PIM-Inh.II & 8 & $26.3 \pm 1.1$ & $75.6 \pm 3.7$ & $0.29 \pm 0.01$ & $23.0 \pm 1.2$ & $10.3 \pm 1.4$ & $13.5 \pm 1.5$ & $30.7 \pm 1.7$ \\
\hline
\end{tabular}

Data are mean \pm SEM.

${ }^{*}$ Significantly $(P<0.05)$ different from CON. ${ }^{\dagger}$ Significantly $(P<0.05)$ different from DES+PIM-Inh.II. ${ }^{\ddagger}$ Significantly $(P<0.05)$ different from IPOST+PIM-Inh.II.

BW, body weight; LV, left ventricle; AAR, area at risk; IS, infarct size; CON, control; DMSO, dimethyl sulfoxide; DES, desflurane; PIM-Inh.II, Pim-1 kinase inhibitor II; IPOST, ischaemic postconditioning; SEM, standard error of the mean.

conditioning, IPOST was completely abolished by application of PIM-Inh.II (IPOST+PIM-Inh.II; $44 \% \pm 5 \%$ ).

\section{Western immunoblotting}

Total Pim-1 kinase protein expression (Fig. 3) as well as total Bad expression (Fig. 4A) was not affected by any of the study drugs. DES did not significantly increase phosphorylation of Bad at serine 112. DESinduced phosphorylation of Bad at serine 112 was significantly reduced by PIM-Inh.II (Fig. 4B). Expression of $\mathrm{Bcl}-2$ protein was not affected by any intervention (Fig. 5).

\section{Discussion}

This study investigated the role of Pim-1 kinase in the signalling cascade of ischaemic and DESinduced postconditioning in a murine in vivo model of acute myocardial infarction. Both 15-min administration of 1 MAC DES during early reperfusion and three cycles of each 10-s reperfusion/ischaemia immediately after prolonged myocardial ischaemia exerted remarkable cardioprotective effects, represented by a pronounced reduction in myocardial IS. These results are in line with findings from various other investigations regarding either APOST ${ }^{5,11,29}$ or 


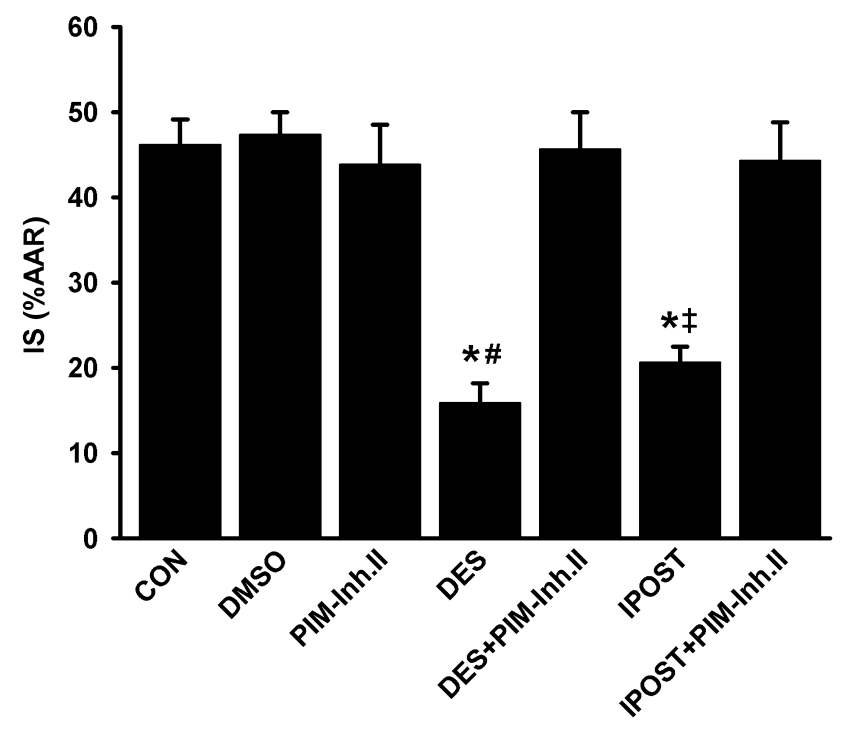

Fig. 2. Cardioprotection by desflurane (DES)-induced and ischaemic postconditioning (IPOST) is completely abolished by pharmacological blockade of Pim-1 kinase. Myocardial infarct size (IS) expressed as percentage of left ventricular area at risk (AAR). Values are mean \pm standard error of the mean. $\mathrm{n}=8$ in each group. * significantly $(\mathrm{P}<0.05)$ different from control $(\mathrm{CON})$ and Pim-1 kinase inhibitor II (PIM-Inh.II). " significantly $(\mathrm{P}<0.05)$ different from DES + PIM-Inh.II. ${ }^{\ddagger}$ Significantly $(\mathrm{P}<0.05)$ different from IPOST+PIM-Inh.II. Mice received either no intervention (CON), dimethyl sulfoxide (DMSO), PIM-Inh.II), DES alone or in combination with PIM-Inh.II (DES+PIM-Inh.II), and IPOST either alone or in combination with PIM-Inh.II (IPOST+PIMInh.II)

IPOST, $^{5-7,29}$ and provide further evidence that APOST and IPOST are promising strategies to alleviate cardiac ischaemia/reperfusion injury. In this study, DES and IPOST reduced myocardial IS to a similar extent. Our results confirm results from other investigations either in rabbits ${ }^{29}$ or mice. ${ }^{5,6}$

A major finding of the present study is that blockade of Pim-1 kinase using the highly selective ${ }^{25}$ PIM-Inh.II completely abolishes DES-induced postconditioning and IPOST against myocardial infarction. The dosage of $10 \mu \mathrm{g} / \mathrm{g}$ body weight has recently been demonstrated to inhibit Pim-1 kinase activity effectively in mice in vivo. ${ }^{21}$ Our results demonstrate a possible role for the serine/threonine kinase Pim-1 in the signalling pathway of APOST and IPOST. Regarding DES, the present results are similar to the previous finding that blockade of Pim-1 kinase completely abolishes DES-induced preconditioning, ${ }^{21}$ indicating a similar signal transduction pathway of DES-induced preconditioning and DES-induced postconditioning. In contrast with IPC that was only partially blocked by Pim-1 inhibi-

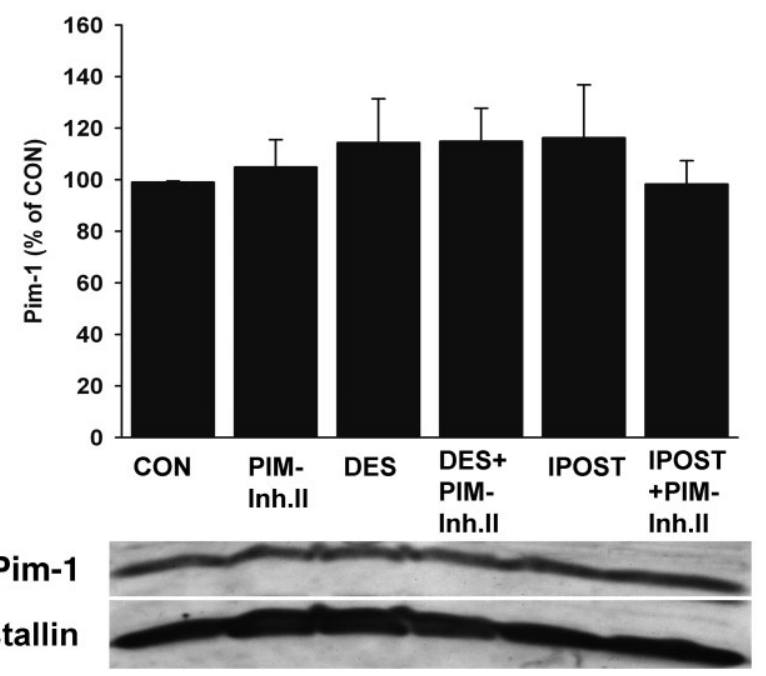

Fig. 3. Myocardial expression of Pim-1 kinase is not affected by DES-induced and IPOST. Western blot analysis of myocardial expression of Pim-1 kinase. Results are presented as representative original immunoblottings and average densitometric results as percentage of control ( $\mathrm{n}=4$ per group). CON, control group; PIMInh.II, Pim-1 kinase inhibitor II (10 $\mu \mathrm{g} / \mathrm{g})$; DES, desflurane (1.0 minimum alveolar concentration); DES+PIM-Inh.II, PIM-Inh.II in combination with DES; IPOST, ischaemic postconditioning (three cycles of each 10-s ischaemia/reperfusion); IPOST+PIMInh.II, PIM-Inh.II in combination with IPOST.

tion, ${ }^{21}$ IPOST is completely abolished by Pim-1 blockade. This might imply differences in the exact role of Pim-1 kinase in the signalling cascades of IPC and IPOST. Further studies are required to reveal these possible differences.

Considerable evidence has accumulated that the serine/threonine kinase Pim-1 exerts relevant cardioprotective properties. Muraski et al. demonstrated a crucial role for Pim-1 in the regulation of cardiomyocyte survival downstream of $\mathrm{Akt}^{18}{ }^{18}$ which itself is a well-known mediator of ischaemic ${ }^{14}$ and $\mathrm{APOST}^{10}$ as a part of the RISK pathway. ${ }^{16}$ Transgenic animals with myocardial overexpression of Pim-1 kinase exhibit less myocardial ISs ${ }^{18}$ and are protected from remodelling following transaortic constriction. ${ }^{19}$ More recently, the same group demonstrated that female mice that receive cardiac progenitor cells overexpressing Pim-1 kinase show improved ventricular function and reduced myocardial IS. $^{20}$ Additionally, Pim-1 kinase increases expression of the sarco/endoplasmatic reticulum $\mathrm{Ca}^{2+}$-adenosine triphosphatase, ${ }^{18}$ which might be involved in the prevention of cardiomyocyte calcium overload during myocardial reperfusion. Furthermore, it has been reported that the highly sequence homologous and structurally similar 

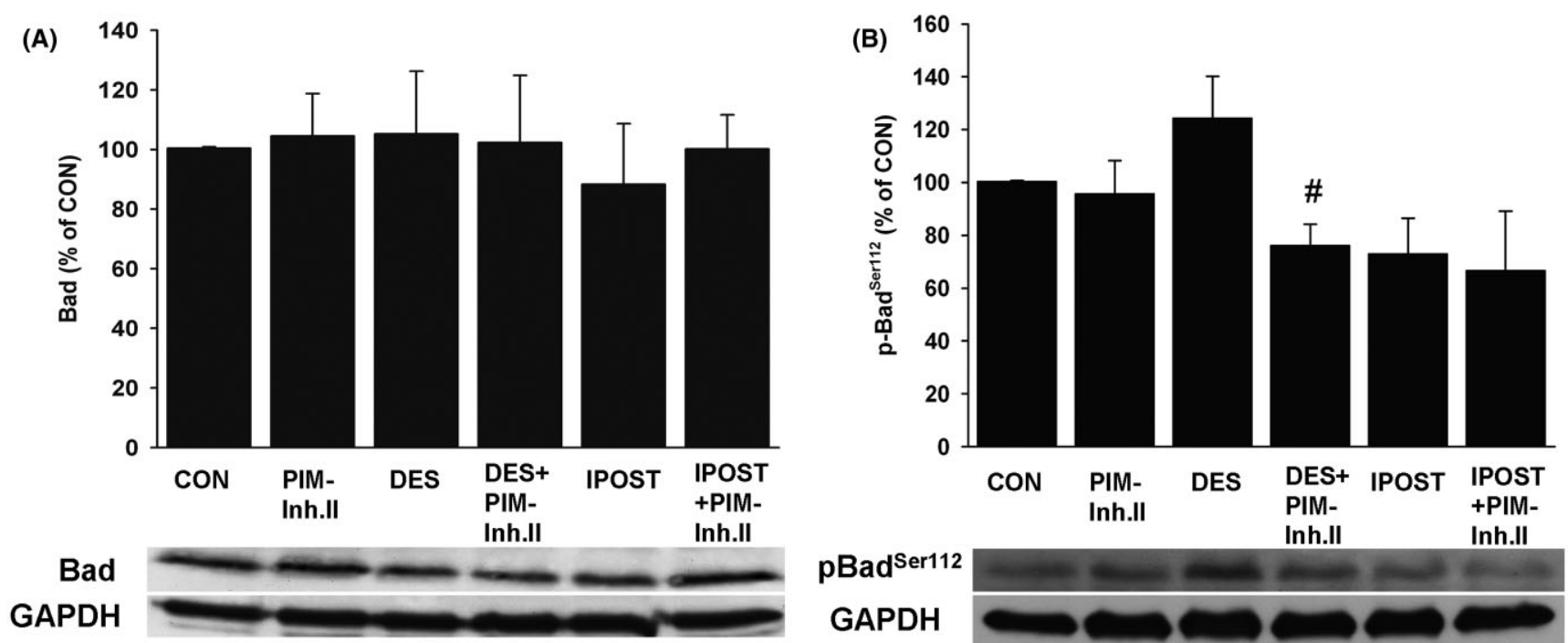

Fig. 4. DES-induced phosphorylation of Bad at serine 112 is reduced by blockade of Pim-1 kinase. Western blot analysis of myocardial expression of $\mathrm{Bad}(\mathrm{A})$ and phospho-Bad ${ }^{\mathrm{Ser112}}(\mathrm{B})$. Results are presented as representative original immunoblottings and average densitometric results as percentage of control $(\mathrm{n}=4$ per group). " significantly $(\mathrm{P}<0.05)$ different from DES. CON, control group; PIM-Inh.II, Pim-1 kinase inhibitor II $(10 \mu \mathrm{g} / \mathrm{g})$; DES, desflurane (1.0 minimum alveolar concentration); DES+PIM-Inh.II, PIM-Inh.II in combination with DES; IPOST, ischaemic postconditioning (three cycles of each 10-s ischaemia/reperfusion); IPOST+PIM-Inh.II, PIM-Inh.II in combination with IPOST.

Pim-3 kinase protects against cardiomyocyte apoptosis after anoxia/re-oxygenation in rat neonatal cardiac myocytes. ${ }^{30}$ Thus, the results of the present study provide further evidence that Pim-1 kinase might be a promising target to alleviate the detrimental effects of cardiac ischaemia/reperfusion injury.

We did not observe differences in Pim-1 expression in whole cell lysates among groups that could have affected our results. These findings confirm results from our previous study. ${ }^{21} \mathrm{~A}$ recent investigation in isolated rat hearts demonstrated a decreased Pim-1 expression in the cytosolic fraction after ischaemia/reperfusion alone. ${ }^{31}$ This decrease was attenuated by IPOST, possibly indicating changes in the intracellular distribution of Pim-1 kinase induced by IPOST. ${ }^{31}$ The differences between both studies might be, most probably, due to different cell fractions that were analysed (cytosolic fraction vs. whole cell lysate) or due to a different experimental model (isolated rat heart vs. in vivo mouse heart), a different I/R protocol (30-min global ischaemia/120-min reperfusion vs. $45-\mathrm{min}$ LAD occlusion/60-min reperfusion) or a different postconditioning protocol (five cycles of each 10-s reperfusion/ischaemia vs. three cycles of each 10-s reperfusion/ischaemia). Thus, our study and

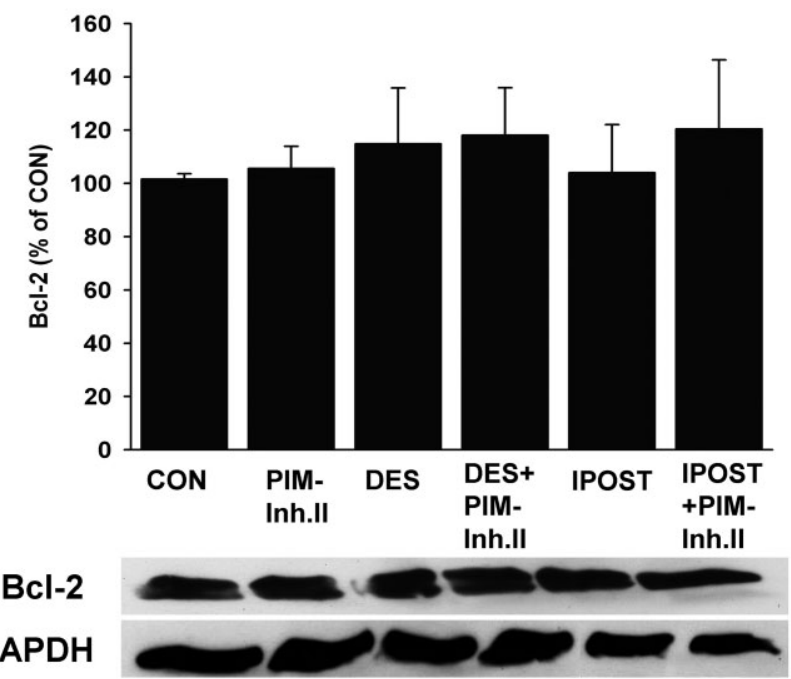

Fig. 5. Expression of B-cell lymphoma 2 (Bcl-2) is not affected by any intervention. Western blot analysis of myocardial expression of $\mathrm{Bcl}-2$. Results are presented as representative original immunoblottings and average densitometric results as percentage of control ( $\mathrm{n}=4$ per group). CON, control group; PIM-Inh.II, Pim-1 kinase inhibitor II $(10 \mu \mathrm{g} / \mathrm{g})$; DES, desflurane (1.0 minimum alveolar concentration); DES+PIM-Inh.II, PIM-Inh.II in combination with DES; IPOST, ischaemic postconditioning (three cycles of each 10-s ischaemia/reperfusion); IPOST+PIM-Inh.II, PIM-Inh.II in combination with IPOST. 


\section{J. Stumpner et al.}

Penna's study ${ }^{31}$ are not directly comparable. More recently, Borillo et al. demonstrated an increase in mitochondrial Pim-1 levels after 30-min ischaemia and 30-min reperfusion, and a corresponding decrease in cytosolic Pim-1 levels after 30-min ischaemia and 10-min reperfusion. ${ }^{32}$

Pim-1 kinase is known to phosphorylate the proapoptotic downstream molecule Bad specifically on residue serine $112 .{ }^{33}$ This phosphorylation in turn results in inactivation of $\mathrm{Bad}^{33} \mathrm{~A}$ direct role for Pim-1 in the prevention of cell death was suggested because inactive Bad enhances Bcl-2 activity, which promotes cell survival. ${ }^{33}$ Transgenic overexpression of Pim-1 kinase in cardiomyocytes has been shown to prevent apoptosis. ${ }^{18}$ In the present study, Pim-1 expression was not different among groups. Therefore, the observed differences in phospho-Bad ${ }^{\mathrm{Ser112}}$ expression can be more likely attributed to different activity levels of Pim-1 kinase rather than different Pim-1 expression levels. Our results demonstrate that DES tends to increase phosphorylation of Bad at serine 112, although this increase did not reach statistical significance. These results are consistent with potential anti-apoptotic properties of the volatile agent. DES-induced phosphorylation of Bad was significantly reduced by pharmacological blockade of Pim-1 kinase. These results imply an involvement of Pim-1 kinase activation in the signalling cascade of DES-induced postconditioning. However, IPOST tends to decrease phosphorylation of Bad at serine 112, which was not affected by blockade of Pim-1 kinase, although this did not reach statistical significance again. PIM-Inh.II alone did not affect phosphorylation of Bad at serine 112. Thus, it is currently not clear whether phosphorylation of Bad at serine 112 by Pim-1 kinase is required for protection by IPOST. Further studies are needed to address this issue.

$\mathrm{Bcl}-2$ protein is a key regulator of the apoptotic cascade and is activated, among others, by phospho$\mathrm{Bad}^{\mathrm{Ser112}}{ }^{33}$ Activation of $\mathrm{Bcl}-2$ prevents Cytochrome $\mathrm{C}$ release into the cytosol, ${ }^{34}$ which is known to be an early event of the apoptotic cascade. ${ }^{35}$ Overexpression of Bcl-2 in cardiac myocytes reduced ischaemia-induced necrosis as well as apoptosis. ${ }^{36}$ Bcl-2 delays opening of the mitochondrial permeability transition pore (mPTP) ${ }^{37}$ A crucial role for mPTP has been demonstrated for APOST ${ }^{38,39}$ and IPOST. $^{40}$ Ischaemic and isoflurane-induced postconditioning are mediated by Bcl-2 in rabbits because pharmacological inhibition of Bcl-2 with HA14-1 abolished IS reduction by short cycles of reperfusion/ischaemia or isoflurane exposure during early reperfusion. ${ }^{29}$ In another study, isoflurane enhanced $\mathrm{Bcl}-2$ expression in neonatal rat cardiac myocytes. ${ }^{41}$ IPC reduced apoptosis by an induction of Bcl-2 gene in isolated rat hearts. ${ }^{42}$ Penna et al. reported a decrease of both cytosolic and mitochondrial Bcl-2 levels by ischaemia/reperfusion alone. ${ }^{31}$ IPOST attenuated this decrease and induced a different redistribution of Bcl-2 between cytosolic and mitochondrial fraction. ${ }^{31}$ Our data demonstrate no differences in Bcl-2 expression among groups. However, this is most probably due to our experimental setting, where myocardial tissue for Western immunoblotting was extracted after $1 \mathrm{~h}$ of reperfusion in contrast to $2 \mathrm{~h}$ in the study by Penna et al. ${ }^{31}$ Taken together with previous results, ${ }^{31,41}$ we suggest that this time, window is too short to detect possible differences in Bcl-2 expression.

The results of the current study should be interpreted within the constraint of several potential limitations. PIM-Inh.II has been demonstrated to be selective to Pim-1 kinase ${ }^{25}$ with high selectivity over Pim-2 kinase. PIM-Inh.II was reported to have a half maximal inhibitory concentration $\left(\mathrm{IC}_{50}\right)$ for Pim-1 kinase of $50 \mathrm{nM}$, whereas the $\mathrm{IC}_{50}$ for Pim-2 kinase was described to be $>20 \mu \mathrm{M} \cdot{ }^{25}$ Nevertheless, potential effects of PIM-Inh.II on Pim-2 and Pim-3 kinase and other kinases involved in the signalling of either APOST or IPOST cannot be completely excluded. We did not measure Pim-1 activity directly. Phosphorylation of Bad at serine 112 was used as a surrogate for Pim-1 activity. Pim-1 kinase has been shown to specifically phosphorylate Bad on serine $112 .{ }^{33}$ However, it cannot be excluded that other kinases involved in postconditioning cascades, particularly Akt, might phosphorylate $\mathrm{Bad}$ on the same residue. However, it has been described that Akt phosphorylates Bad predominantly at serine $136 .^{43}$

Taken together, our results demonstrate that both DES-induced postconditioning and IPOST against myocardial infarction are mediated by the serine/ threonine kinase Pim-1. DES-induced postconditioning might induce some anti-apoptotic effects, at least in part depending on Pim-1 activity. Thus, the present findings further support the concept that targeting anti-apoptotic proteins might be beneficial in the treatment of myocardial ischaemia/ reperfusion injury.

\section{Acknowledgements}

The authors thank Katerina Pech, Department of Anaesthesia and Critical Care, University of Würzburg, for excellent technical assistance. 
Funding: Funding was provided from institutional/ departmental sources only.

Conflicts of interest: Franz Kehl previously received honoraria for lectures and investigator-initiated trials from Baxter, Germany. The other authors state no conflict of interest.

\section{References}

1. Braunwald E, Kloner RA. Myocardial reperfusion - a double-edged sword. J Clin Invest 1985; 76: 1713-9.

2. Yellon DM, Hausenloy DJ. Myocardial reperfusion injury. $N$ Engl J Med 2007; 357: 1121-35.

3. Zhao ZQ, Corvera JS, Halkos ME, Kerendi F, Wang NP, Guyton RA, Vinten-Johansen J. Inhibition of myocardial injury by ischemic postconditioning during reperfusion: comparison with ischemic preconditioning. Am J Physiol Heart Circ Physiol 2003; 285: H579-H88.

4. Argaud L, Gateau-Roesch O, Augeul L, Couture-Lepetit E, Loufouat J, Gomez L, Robert D, Ovize M. Increased mitochondrial calcium coexists with decreased reperfusion injury in postconditioned (but not preconditioned) hearts. Am J Physiol Heart Circ Physiol 2008; 294: H386-H91.

5. Redel A, Stumpner J, Tischer-Zeitz T, Lange M, Smul TM, Lotz C, Roewer N, Kehl F. Comparison of isoflurane-, sevoflurane-, and desflurane-induced pre- and postconditioning against myocardial infarction in mice in vivo. Exp Biol Med (Maywood) 2009; 234: 1186-91.

6. Tsutsumi YM, Yokoyama T, Horikawa Y, Roth DM, Patel $\mathrm{HH}$. Reactive oxygen species trigger ischemic and pharmacological postconditioning: in vivo and in vitro characterization. Life Sci 2007; 81: 1223-7.

7. Boengler K, Buechert A, Heinen Y, Roeskes C, HilfikerKleiner D, Heusch G, Schulz R. Cardioprotection by ischemic postconditioning is lost in aged and STAT3deficient mice. Circ Res 2008; 102: 131-5.

8. Thibault H, Piot C, Staat P, Bontemps L, Sportouch C, Rioufol G, Cung TT, Bonnefoy E, Angoulvant D, Aupetit JF, Finet G, Andre-Fouet X, Macia JC, Raczka F, Rossi R, Itti R, Kirkorian G, Derumeaux G, Ovize M. Long-term benefit of postconditioning. Circulation 2008; 117: 1037-44.

9. Schlack W, Preckel B, Barthel H, Obal D, Thamer V. Halothane reduces reperfusion injury after regional ischaemia in the rabbit heart in vivo. Br J Anaesth 1997; 79: 88-96.

10. Chiari PC, Bienengraeber MW, Pagel PS, Krolikowski JG, Kersten JR, Warltier DC. Isoflurane protects against myocardial infarction during early reperfusion by activation of phosphatidylinositol-3-kinase signal transduction: evidence for anesthetic-induced postconditioning in rabbits. Anesthesiology 2005; 102: 102-9.

11. Lange M, Redel A, Lotz C, Smul TM, Blomeyer C, Frank A, Stumpner J, Roewer N, Kehl F. Desflurane-induced postconditioning is mediated by beta-Adrenergic signaling: role of beta1- and beta2-Adrenergic receptors, protein kinase $\mathrm{A}$, and calcium/calmodulin-dependent protein kinase II. Anesthesiology 2009; 110: 516-28.

12. Deyhimy DI, Fleming NW, Brodkin IG, Liu H. Anesthetic preconditioning combined with postconditioning offers no additional benefit over preconditioning or postconditioning alone. Anesth Analg 2007; 105: 316-24.

13. Lemoine S, Beauchef G, Zhu L, Renard E, Lepage O, Massetti M, Khayat A, Galera P, Gerard JL, Hanouz JL. Signaling pathways involved in desflurane-induced postconditioning in human atrial myocardium in vitro. Anesthesiology 2008; 109: 1036-44.

14. Tsang A, Hausenloy DJ, Mocanu MM, Yellon DM. Postconditioning: a form of 'modified reperfusion' protects the myo- cardium by activating the phosphatidylinositol 3-kinase-Akt pathway. Circ Res 2004; 95: 230-2.

15. Li H, Wang JK, Zeng YM, Yang CX, Chen HT, Wen XJ, Shui CL, Liang H. Sevoflurane post-conditioning protects against myocardial reperfusion injury by activation of phosphatidylinositol-3-kinase signal transduction. Clin Exp Pharmacol Physiol 2008; 35: 1043-51.

16. Hausenloy DJ, Yellon DM. Reperfusion injury salvage kinase signalling: taking a RISK for cardioprotection. Heart Fail Rev 2007; 12: 217-34.

17. Bachmann M, Moroy T. The serine/threonine kinase Pim-1. Int J Biochem Cell Biol 2005; 37: 726-30.

18. Muraski JA, Rota M, Misao Y, Fransioli J, Cottage C, Gude N, Esposito G, Delucchi F, Arcarese M, Alvarez R, Siddiqi S, Emmanuel GN, Wu W, Fischer K, Martindale JJ, Glembotski CC, Leri A, Kajstura J, Magnuson N, Berns A, Beretta RM, Houser SR, Schaefer EM, Anversa P, Sussman MA. Pim-1 regulates cardiomyocyte survival downstream of Akt. Nat Med 2007; 13: 1467-75.

19. Muraski JA, Fischer KM, Wu W, Cottage CT, Quijada P, Mason M, Din S, Gude N, Alvarez R Jr, Rota M, Kajstura J, Wang Z, Schaefer E, Chen X, MacDonnel S, Magnuson N, Houser SR, Anversa P, Sussman MA. Pim-1 kinase antagonizes aspects of myocardial hypertrophy and compensation to pathological pressure overload. Proc Natl Acad Sci U S A 2008; 105: 13889-94.

20. Fischer KM, Cottage CT, Wu W, Din S, Gude NA, Avitabile D, Quijada P, Collins BL, Fransioli J, Sussman MA. Enhancement of myocardial regeneration through genetic engineering of cardiac progenitor cells expressing Pim-1 kinase. Circulation 2009; 120: 2077-87.

21. Stumpner J, Redel A, Kellermann A, Lotz CA, Blomeyer CA, Smul TM, Kehl F, Roewer N, Lange M. Differential role of Pim-1 kinase in anesthetic-induced and ischemic preconditioning against myocardial infarction. Anesthesiology 2009; 111: 1257-64.

22. Bayne K. Revised guide for the care and use of laboratory animals available. American Physiological Society. Physiologist 1996; 39: 208-11.

23. Redel A, Jazbutyte V, Smul TM, Lange M, Eckle T, Eltzschig $\mathrm{H}$, Roewer N, Kehl F. Impact of ischemia and reperfusion times on myocardial infarct size in mice in vivo. Exp Biol Med (Maywood) 2008; 233: 84-93.

24. Eckle T, Grenz A, Kohler D, Redel A, Falk M, Rolauffs B, Osswald H, Kehl F, Eltzschig HK. Systematic evaluation of a novel model for cardiac ischemic preconditioning in mice. Am J Physiol Heart Circ Physiol 2006; 291: H2533-H40.

25. Cheney IW, Yan S, Appleby $T$, Walker $H$, Vo $T$, Yao N, Hamatake R, Hong Z, Wu JZ. Identification and structure-activity relationships of substituted pyridones as inhibitors of Pim-1 kinase. Bioorg Med Chem Lett 2007; 17: 1679-83.

26. Sonner JM, Gong D, Eger EI. Naturally occurring variability in anesthetic potency among inbred mouse strains. Anesth Analg 2000; 91: 720-6.

27. Kin H, Zhao ZQ, Sun HY, Wang NP, Corvera JS, Halkos ME, Kerendi F, Guyton RA, Vinten-Johansen J. Postconditioning attenuates myocardial ischemia-reperfusion injury by inhibiting events in the early minutes of reperfusion. Cardiovasc Res 2004; 62: 74-85.

28. Redel A, Lange M, Jazbutyte V, Lotz C, Smul TM, Roewer N, Kehl F. Activation of mitochondrial large-conductance calcium-activated $\mathrm{K}+$ channels via protein kinase A mediates desflurane-induced preconditioning. Anesth Analg 2008; 106: 384-91.

29. Wang C, Neff DA, Krolikowski JG, Weihrauch D, Bienengraeber M, Warltier DC, Kersten JR, Pagel PS. The influence 


\section{J. Stumpner et al.}

of B-cell lymphoma 2 protein, an antiapoptotic regulator of mitochondrial permeability transition, on isofluraneinduced and ischemic postconditioning in rabbits. Anesth Analg 2006; 102: 1355-60.

30. Liu D, He M, Yi B, Guo WH, Que AL, Zhang JX. Pim-3 protects against cardiomyocyte apoptosis in anoxia/ reoxygenation injury via p38-mediated signal pathway. Int J Biochem Cell Biol 2009; 41: 2315-22.

31. Penna C, Perrelli MG, Raimondo S, Tullio F, Merlino A, Moro F, Geuna S, Mancardi D, Pagliaro P. Postconditioning induces an anti-apoptotic effect and preserves mitochondrial integrity in isolated rat hearts. Biochim Biophys Acta 2009; 1787: 794-801.

32. Borillo GA, Mason M, Quijada P, Volkers M, Cottage C, McGregor M, Din S, Fischer K, Gude N, Avitabile D, Barlow S, Alvarez R, Truffa S, Whittaker R, Glassy MS, Gustafsson AB, Miyamoto S, Glembotski CC, Gottlieb RA, Brown JH, Sussman MA. Pim-1 kinase protects mitochondrial integrity in cardiomyocytes. Circ Res 2010; 106: 1265-74.

33. Aho TL, Sandholm J, Peltola KJ, Mankonen HP, Lilly M, Koskinen PJ. Pim-1 kinase promotes inactivation of the proapoptotic Bad protein by phosphorylating it on the Ser112 gatekeeper site. FEBS Lett 2004; 571: 43-9.

34. Kluck RM, Bossy-Wetzel E, Green DR, Newmeyer DD. The release of cytochrome $\mathrm{c}$ from mitochondria: a primary site for Bcl-2 regulation of apoptosis. Science 1997; 275: 1132-6.

35. Bossy-Wetzel E, Newmeyer DD, Green DR. Mitochondrial cytochrome c release in apoptosis occurs upstream of DEVD-specific caspase activation and independently of mitochondrial transmembrane depolarization. EMBO J 1998; 17: 37-49.

36. Imahashi K, Schneider MD, Steenbergen C, Murphy E. Transgenic expression of Bcl-2 modulates energy metabolism, prevents cytosolic acidification during ischemia, and reduces ischemia/reperfusion injury. Circ Res 2004; 95: 73441.

37. Zhu L, Yu Y, Chua BH, Ho YS, Kuo TH. Regulation of sodium-calcium exchange and mitochondrial energetics by Bcl-2 in the heart of transgenic mice. J Mol Cell Cardiol 2001; 33: $2135-44$.
38. Feng J, Lucchinetti E, Ahuja P, Pasch T, Perriard JC, Zaugg $\mathrm{M}$. Isoflurane postconditioning prevents opening of the mitochondrial permeability transition pore through inhibition of glycogen synthase kinase 3beta. Anesthesiology 2005; 103: 987-95.

39. Ge ZD, Pravdic D, Bienengraeber M, Pratt PF Jr, Auchampach JA, Gross GJ, Kersten JR, Warltier DC. Isoflurane postconditioning protects against reperfusion injury by preventing mitochondrial permeability transition by an endothelial nitric oxide synthase-dependent mechanism. Anesthesiology 2010; 112: 73-85.

40. Argaud L, Gateau-Roesch O, Raisky O, Loufouat J, Robert D, Ovize M. Postconditioning inhibits mitochondrial permeability transition. Circulation 2005; 111: 194-7.

41. Jamnicki-Abegg M, Weihrauch D, Pagel PS, Kersten JR, Bosnjak ZJ, Warltier DC, Bienengraeber MW. Isoflurane inhibits cardiac myocyte apoptosis during oxidative and inflammatory stress by activating Akt and enhancing Bcl-2 expression. Anesthesiology 2005; 103: 1006-14.

42. Maulik N, Engelman RM, Rousou JA, Flack JE 3rd, Deaton $\mathrm{D}$, Das DK. Ischemic preconditioning reduces apoptosis by upregulating anti-death gene Bcl-2. Circulation 1999; 100: II369-75.

43. Datta SR, Dudek H, Tao X, Masters S, Fu H, Gotoh Y, Greenberg ME. Akt phosphorylation of BAD couples survival signals to the cell-intrinsic death machinery. Cell 1997; 91: 231-41.

Address:

Jan Stumpner

Universität Würzburg

Klinik und Poliklinik für Anästhesiologie, Zentrum Operative

Medizin

Oberdürrbacher Street 6

97080 Würzburg

Germany

e-mail: stumpner_j@klinik.uni-wuerzburg.de 